\title{
ArcheoSciences
}

Revue d'archéométrie

\section{La production monétaire romaine en orichalque : caractérisation du monnayage et approche du processus d'élaboration par l'expérimentation}

Roman Monetary Production in Orichalcum: Characterization of Coinage and Approach of Working-off Process by Experimentation

Gaffiero, Florian Téreygeol, A. Suspène, Bernard Gratuze et S. Zeller

\section{OpenEdition}

\section{Journals}

Édition électronique

URL : https://journals.openedition.org/archeosciences/2951

DOI : 10.4000/archeosciences.2951

ISBN : 978-2-7535-1849-0

ISSN : 2104-3728

Éditeur

Presses universitaires de Rennes

Édition imprimée

Date de publication : 30 avril 2011

Pagination : 93-102

ISBN : 978-2-7535-1847-6

ISSN : 1960-1360

Référence électronique

Gaffiero, Florian Téreygeol, A. Suspène, Bernard Gratuze et S. Zeller, «La production monétaire romaine en orichalque : caractérisation du monnayage et approche du processus d'élaboration par l'expérimentation », ArcheoSciences [En ligne], 35 | 2011, mis en ligne le 30 avril 2013, consulté le 28 février 2022. URL : http://journals.openedition.org/archeosciences/2951 ; DOI : https://doi.org/ 10.4000/archeosciences.2951

Ce document a été généré automatiquement le 28 février 2022.

Article L.111-1 du Code de la propriété intellectuelle. 


\section{La production monétaire romaine en orichalque : caractérisation du monnayage et approche du processus d'élaboration par l'expérimentation}

Roman Monetary Production in Orichalcum: Characterization of Coinage and Approach of Working-off Process by Experimentation

Gaffiero, Florian Téreygeol, A. Suspène, Bernard Gratuze et S. Zeller

\section{Introduction}

1 Cet article rassemble les premiers résultats obtenus au cours d'une étude du monnayage romain en orichalque, et concerne plus particulièrement l'élaboration de l'orichalque utilisé comme alliage monétaire.

2 Le monnayage romain en orichalque apparaît vers $23 \mathrm{av.J.-C}$. sous le principat d'Auguste. En effet, Auguste (le fils adoptif de Jules César, également connu sous les noms d'Octave et d'Octavien) créé un nouveau système monétaire constitué de quatre métaux et alliages différents : les monnaies d'or (les aurei), les monnaies d'argent (les deniers), les monnaies en orichalque (les sesterces, dupondii et semisses) et les monnaies de cuivre (les as et quadrantes). Cet ensemble ne disparaîtra qu'en 294 lors des réformes monétaires de Dioclétien (Burnett, 1988), (Christol et Nony, 2003).

3 Sous l'Empire, les alliages monétaires subirent différentes modifications. Si le titre des monnaies d'or ne changea quasiment pas (Callu et al., 1985), les deniers furent dévalués régulièrement (Walker, 1976), (Walker, 1977) et (Walker, 1978). Afin d'émettre plus de monnaies en période de crise, on augmentait la part de cuivre présente dans l'alliage pour avoir une quantité de métal plus importante à moindre coût. 
4 Après avoir analysé différentes monnaies de l'empire romain, Caley a le premier montré qu'il en est de même pour le monnayage en orichalque (Caley, 1964). La part de zinc dans les sesterces, dupondii et semisses d'orichalque (ou laiton) évolue. Elle semble diminuer régulièrement.

5 Trois hypothèses ont été émises pour expliquer cette baisse régulière de la teneur en zinc.

6 - Les monnaies des empereurs précédents seraient refondues pour servir de matière première aux nouvelles monnaies. Or le zinc est l'élément le plus instable : il est volatil aux températures nécessaires à la refonte du laiton. Il serait donc logique que l'on observe une baisse régulière de la teneur en zinc dans les monnaies au cours des émissions. Il disparaît de l'alliage en se vaporisant, puis s'oxyde en réagissant immédiatement avec l'oxygène de l'air présent dans le creuset et le four.

7 - L'utilisation d'un laiton avec une teneur en zinc différente ou/et l'ajout de cuivre au laiton lors de la fabrication des monnaies en orichalque pourraient-ils aussi expliquer la baisse régulière de la teneur en zinc? L'adjonction de cuivre pourrait diminuer la teneur en zinc tout en permettant une production accrue de monnaie, comme on l'observe pour le monnayage d'argent (Burnett, 1988).

8 - Enfin, il pourrait aussi y avoir une combinaison des deux premières hypothèses, soit des refontes monétaires ponctuelles ou systématiques avec ajout d'un alliage provenant d'une source différente (ajout de cuivre ou d'un autre laiton). De cette façon, on obtiendrait plus de métal pour fabriquer des nouvelles monnaies tout en conservant suffisamment de monnaies en circulation.

9 Les deux dernières hypothèses pourraient suggérer une volonté de l'autorité émettrice de diminuer la teneur en zinc tant par manque de métal monétaire que par souci d'économie ${ }^{1}$, tandis que l'hypothèse des seules refontes semble impliquer un contrôle limité de la composition de l'alliage de la part des artisans métallurgistes. S'ils maîtrisent les réactions de l'alliage selon les contraintes subies (ici thermiques), alors une diminution de la teneur en zinc dans les monnaies romaines proviendrait d'une volonté propre. Cette dernière a pu être dictée par un manque de matière première. Dans ce cas, l'utilisation d'un nouvel alliage moins riche en zinc, ou l'ajout de cuivre, permettent de faire face aux besoins. L'évolution de l'alliage peut aussi être l'effet d'une décision politique ou administrative. Il est alors nécessaire que les artisans disposent du savoir-faire approprié. Ainsi, au troisième siècle, le sesterce atteignit une valeur intrinsèque plus importante que celle du denier ; l'autorité politique décida alors l'arrêt définitif de l'emploi du laiton dans le monnayage (Burnett, 1988).

La diminution de la teneur en zinc observée sur les monnaies romaines par E. Caley Caley, 1964) et D. Dungworth (Dungworth, 1996) a été mise en évidence à partir d'un corpus monétaire limité. Afin de vérifier cette tendance, de nouvelles analyses par activation avec des neutrons rapides de cyclotron ont été effectuées ${ }^{2}$ sur les monnaies en orichalque d'Auguste à Dioclétien provenant du Cabinet des Médailles de la Bibliothèque nationale de France. Cette méthode globale permet de détecter aussi bien les éléments majeurs (ici le cuivre et le zinc) que les éléments mineurs inférieurs au pourcent (l'étain et le plomb). De plus, elle a des limites de détection de l'ordre du ppm, ce qui nous donne accès au dosage de certains éléments-traces (tel que l'or, l'antimoine, l'argent, le cobalt, le nickel ou le fer). Nous pouvons ainsi déterminer différents types de minerais ayant pu être employés par les Romains lors de la fabrication des 
monnaies ${ }^{3}$. Ces éléments-traces (Gaffiero et al., à paraitre) permettent aussi de voir si l'hypothèse des refontes est pertinente ou non. En effet, si pour chaque empereur, l'utilisation de minerais de cuivre et (ou) de zinc différents est mise en évidence, alors l'hypothèse des refontes ne peut pas être retenue.

La méthode employée est de plus non destructive (Beauchesne, 1986), ce qui fait que nous sommes à même d'analyser un plus grand nombre de monnaies et ainsi d'affiner la courbe obtenue à partir des données de E. Caley (Figure 2).

Les analyses par voie humide effectuées par Caley impliquaient la destruction de l'objet, ce qui limitait le corpus de l'étude.

Dans un second temps, l'étude de la technique de refonte est prise en considération. Si ce procédé est mieux appréhendé, la détermination de l'hypothèse la plus pertinente pour expliquer l'évolution de l'alliage monétaire des monnaies en orichalque sera plus aisée. L'archéologie expérimentale semble une démarche appropriée pour cette étude : les gestes, les outils et l'environnement des «fondeurs» ne sont plus seulement hypothétiques, ils deviennent tangibles. Ces deux approches combinées permettent alors de mieux comprendre le système monétaire et surtout les procédés de fabrication.

\section{Corpus et méthode d'analyse}

Dans cette étude, nous avons donc été amenés à analyser d'une part des monnaies romaines d'Auguste à Antonin le Pieux (fig. 1), et d'autre part les flans expérimentaux que nous avons créés.

Figure 1 : Sesterce d'Hadrien (BnF, FG1757).

Figure 1: Hadrian's sestertius (BnF, FG1757).

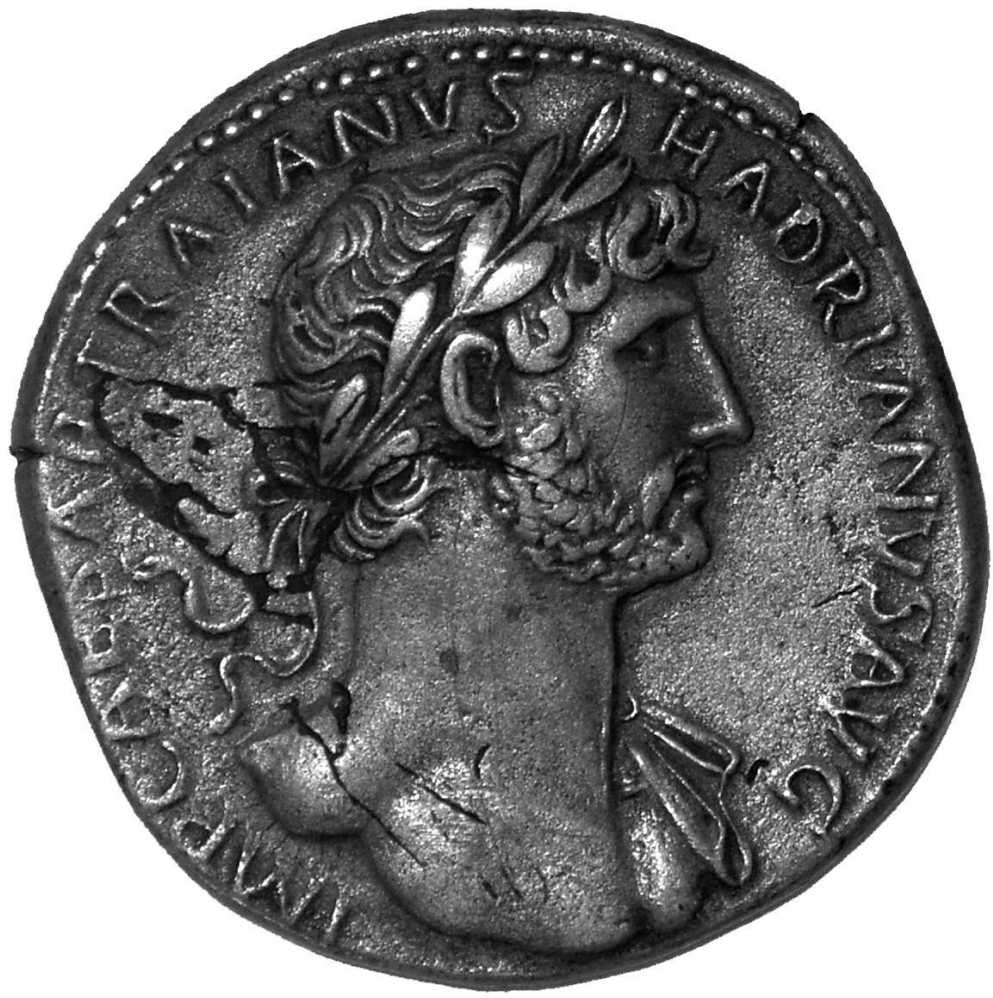




\section{Les monnaies de l'empire romain}

Pour chaque empereur ${ }^{4}$, entre 30 et 60 monnaies $^{5}$ ont été analysées par activation avec des neutrons rapides de cyclotron couplé à la spectrométrie gamma. Des analyses antérieures effectuées à l'IRAMAT d'Orléans par spectrométrie de fluorescence X par J.N. Barrandon sur les monnaies du Trésor de Garonne (Etienne et Rachet, 1984) sont aussi prises en compte dans cette étude. Les monnaies étant en excellent état, les analyses de composition par spectrométrie de fluorescence $\mathrm{X}$ ont été rendu possibles et pertinentes. Ainsi le corpus monétaire des empereurs est considérablement augmenté (jusqu'à 400 monnaies analysées pour certains empereurs).

L'analyse par activation avec des neutrons rapides de cyclotron ${ }^{6}$ (effectuée au laboratoire du CEMTHI d'Orléans) couplé à la spectrométrie gamma (à l'IRAMAT d'Orléans) ne nécessite pas de prélèvement et permet donc d'analyser la monnaie dans sa globalité, en s'affranchissant des problèmes liés à l'hétérogénéité des alliages, ainsi qu'à l'état de surface liés à l'oxydation et au temps d'enfouissement des monnaies. Les limites de détection sont de l'ordre de la partie par million (ppm) pour la plupart des éléments, ce qui donne accès aux teneurs en éléments majeurs (zinc et cuivre ici), mineurs (étain et plomb) et traces (antimoine, argent, arsenic, cobalt, fer, nickel et or). Afin d'obtenir des résultats de mesure les plus fiable possible, nous avons effectué deux à trois comptage sur chaque pièce. La précision obtenue pour les teneurs est de l'ordre de 1 à $5 \%$ relatif pour les éléments majeurs et mineurs et de l'ordre de $10 \%$ relatif pour les éléments traces.

7 Les teneurs qui sont représentées par la suite sur le graphique de la figure 2 sont les teneurs moyennes par empereur accompagnées de leurs écart-types. Ces derniers montrent la variabilité des teneurs selon les empereurs.

Figure 2 : Évolution de la teneur moyenne en zinc présente dans le monnayage romain (analyses obtenues par A. Gaffiero et J. -N. Barrandon par ANRC et spectrométrie de fluorescence X sur le monnayage du trésor de Garonne. Il y a superposition des analyses de E. Caley (Caley, 1964).) Figure 2: Evolution of the average content in zinc in roman's coinage (Analysis were got by A. Gaffiero and J.-N. Barrandon by activation with fast neutrons from a cyclotron and by $X$-ray fluorescence spectroscopy on the "trésor de Garonne" coinage. Superimposed, there are E. Caley's analysis (Caley, 1964).)

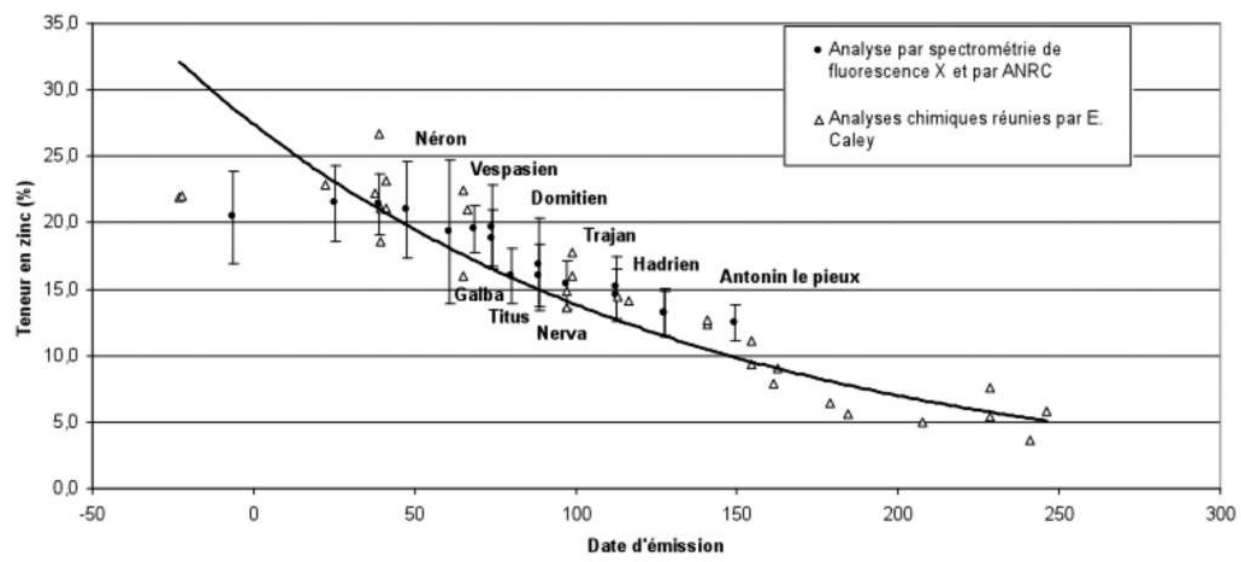




\section{Les flans expérimentaux}

de chaque coulée. Les autres ont été aussitôt refondus afin de continuer l'expérience. Ils ont ensuite été polis pour être analysés par spectrométrie de fluorescence $\mathrm{X}$ à l'IRAMAT d'Orléans. Chaque prélèvement a subi entre 2 (pour les plus petits) et 6 analyses de composition. En multipliant les analyses, nous avons remarqué que les flans ont un alliage homogène. Les teneurs des flans représentées sur les graphiques correspondent donc à la moyenne des teneurs obtenues lors des différentes mesures. L'incertitude sur les teneurs en zinc est de l'ordre de $1 \%$. Les barres d'erreurs ne sont pas représentées afin de rendre le graphique plus lisible. Seuls les écart-types sur les teneurs moyennes sont pris en compte. Ces derniers sont inférieurs à $0,5 \%$, et sont confondus avec les points sur le graphique. Seuls les éléments majeurs (cuivre et zinc) ont été analysés lors des mesures par spectrométrie de fluorescence $\mathrm{X}$. Le protocole de mesure utilisé pour la fluorescence $\mathrm{X}$ ne permettait pas, en effet, la détection des éléments traces présent dans notre alliage expérimental. Les teneurs de ces derniers, mesurées par ANRC sont inférieures à $0,1 \%$ et sont en deçà des limites de détection du protocole utilisé pour les mesures par fluorescence X. De plus, les quelques mesures effectuées par ANRC sur les flans expérimentaux, montrent que les teneurs de ces éléments ne varient pas au fil des refontes et n'interfèrent donc pas avec le cuivre et le zinc. En raison du coût élevé et de la lenteur des analyses par ANRC, l'analyse par spectrométrie de fluorescence $\mathrm{X}$, plus rapide et plus précise, a été choisie pour doser systématiquement le cuivre et le zinc présents dans les flans issus des refontes expérimentales.

\section{L'analyse élémentaire des monnaies}

19 Les travaux de E. Caley dans les années soixante ont révélé que la teneur en zinc présente dans les monnaies impériales diminue au cours du temps (fig. 2). Les analyses de composition chimique par activation avec des neutrons rapides de cyclotron sur un corpus monétaire plus conséquent (400 monnaies) que celui dont disposait Caley montrent une diminution moins nette de la teneur en zinc présente dans les monnaies (fig. 2).

20 Sur ce graphique, la valeur moyenne de la teneur en zinc sous chaque empereur est observée. Celle-ci, représentée avec son écart type qui est relativement important pour chacun des empereurs, montre une relative stabilité de la composition de l'alliage jusqu'à Néron. Ce n'est qu'à partir de Vespasien qu'il y aurait une baisse sensible de la teneur en zinc : l'hypothèse que des refontes seules expliqueraient ce changement n'est déjà plus recevable pour ce monnayage. Si l'écart type maximal de la teneur en zinc pour les monnaies de Domitien est pris en compte, on retrouve pour cet empereur certaines monnaies avec des teneurs en zinc comparables à celles des empereurs précédents. Sous Trajan, la baisse se poursuit, cependant on retrouve toujours des teneurs en zinc propres au monnayage précédent. Cette baisse semble se maintenir par la suite; des analyses par activation avec des neutrons rapides de cyclotron du monnayage des empereurs suivants sont en cours pour vérification.

21 De façon plus générale, il semble qu'il y ait bien une diminution globale de la teneur en zinc dans le monnayage en orichalque romain et l'hypothèse qui implique la refonte 
des monnaies des empereurs précédents pourrait paraître justifiée. Mais elle commencerait seulement après la mort de Néron.

Pour appréhender cette théorie, la reconstitution de la chaîne opératoire permettant l'élaboration de l'alliage monétaire est nécessaire: il faut alors déterminer l'environnement et le matériel qui sera employé pour mener à bien l'expérience. Les différents outils (pinces, creusets, couvercle), un four, des moules et le métal devront être choisis et élaborés. L'archéologie expérimentale est ici le moyen d'approche qui semble le plus pertinent. Les sources antiques sont en effet insuffisantes pour confirmer l'hypothèse d'une diminution de la teneur en zinc liée aux seules refontes, et nous devons donc diversifier les moyens d'investigation pour créer un faisceau de présomptions permettant de proposer des conjectures plus fiables.

\section{Réalisation expérimentale de l'alliage monétaire}

\section{Dispositif expérimental et matière première}

La fabrication monétaire est très peu référencée de façon générale, aussi bien en ce qui concerne la fabrication de l'alliage et la frappe, qu'en ce qui concerne la quantité de monnaies émises pour chaque empereur ${ }^{8}$. Les sources font référence à des changements monétaires aux moyens d'actes techniques tels que les refontes; on trouve une première mention sous le principat de Caligula ${ }^{9}$ et une seconde sous Trajan (Besombes, 2008). Il n'y a pas de traité antique concernant la fabrication et l'outillage. Nous avons dû prendre en compte les traces archéologiques laissées par les ateliers clandestins du III e siècle apr. J.-C. (Pilon, 2002), ainsi que les traces d'activités métallurgiques dans l'Antiquité (Pernot, 1999) pour mettre en place un protocole permettant d'effectuer des refontes dans des conditions proches de ce qu'elles ont pu être. Nous nous sommes aussi inspirés de structures plus récentes mais relativement simples pour l'élaboration du four.

L'équipement qui a été adopté est le suivant.

À partir des données archéologiques du site de Châteaubleau (Pilon, 2002), des moules bivalve en calcaire (fig. 3) sont recréés. 
Figure 3 : moule bivalve en calcaire. Figure 3: Bivalve mould in limestone.

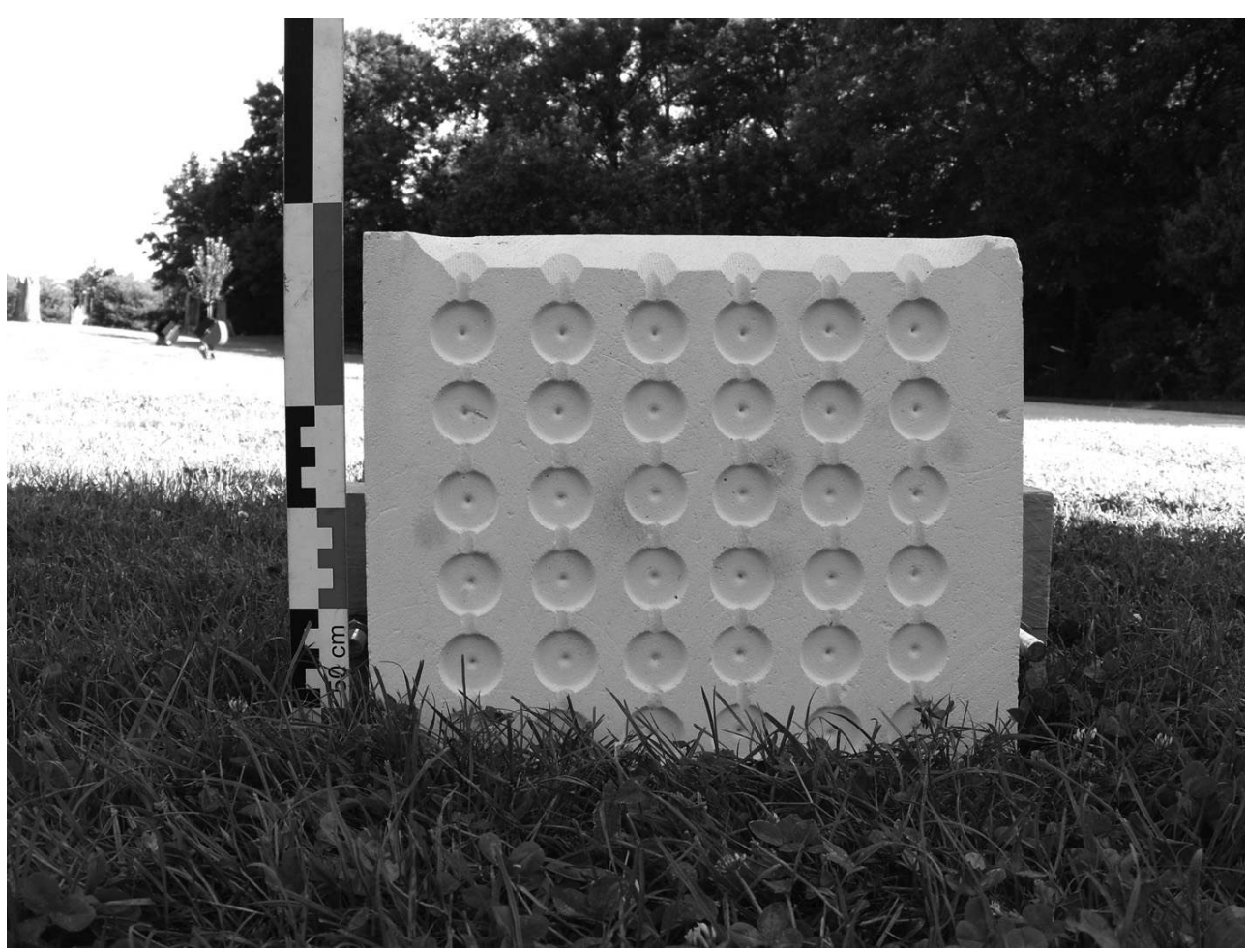

Un couvercle en terre cuite (fig.5) est aussi utilisé pour recouvrir le creuset en plombagine (fig. 4) nécessaire pour la refonte (un creuset en terre cuite n'a pas été choisi lors de cette expérimentation car nous voulions avant tout améliorer l'étape de fusion avant d'employer une matière plus sensible aux différences de température). 
Figure 4 : Creuset plombagine.

Figure 4: Graphite crucible.

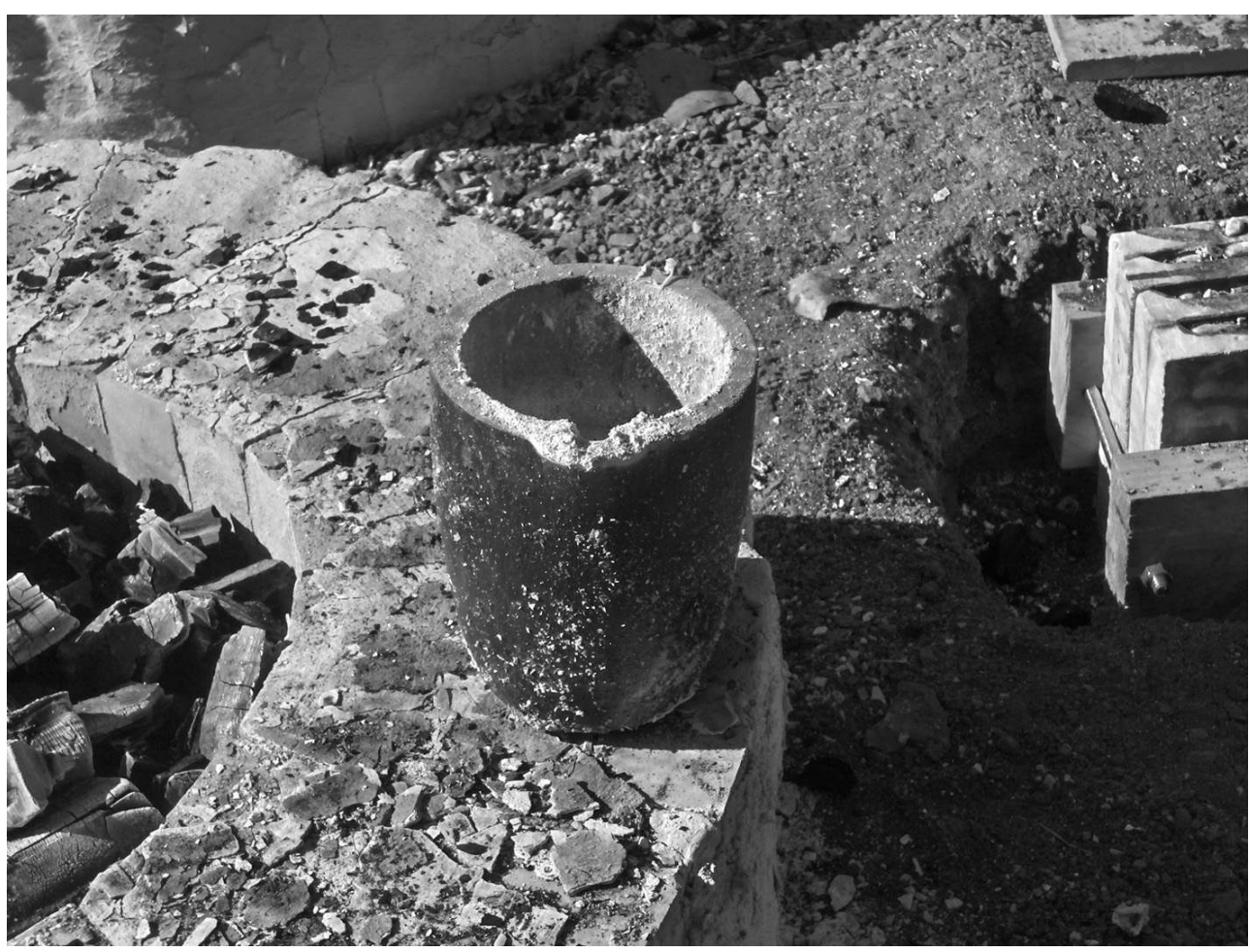

Figure 5 : Four en trou de serrure.

Figure 5: "Keyhole" furnace.

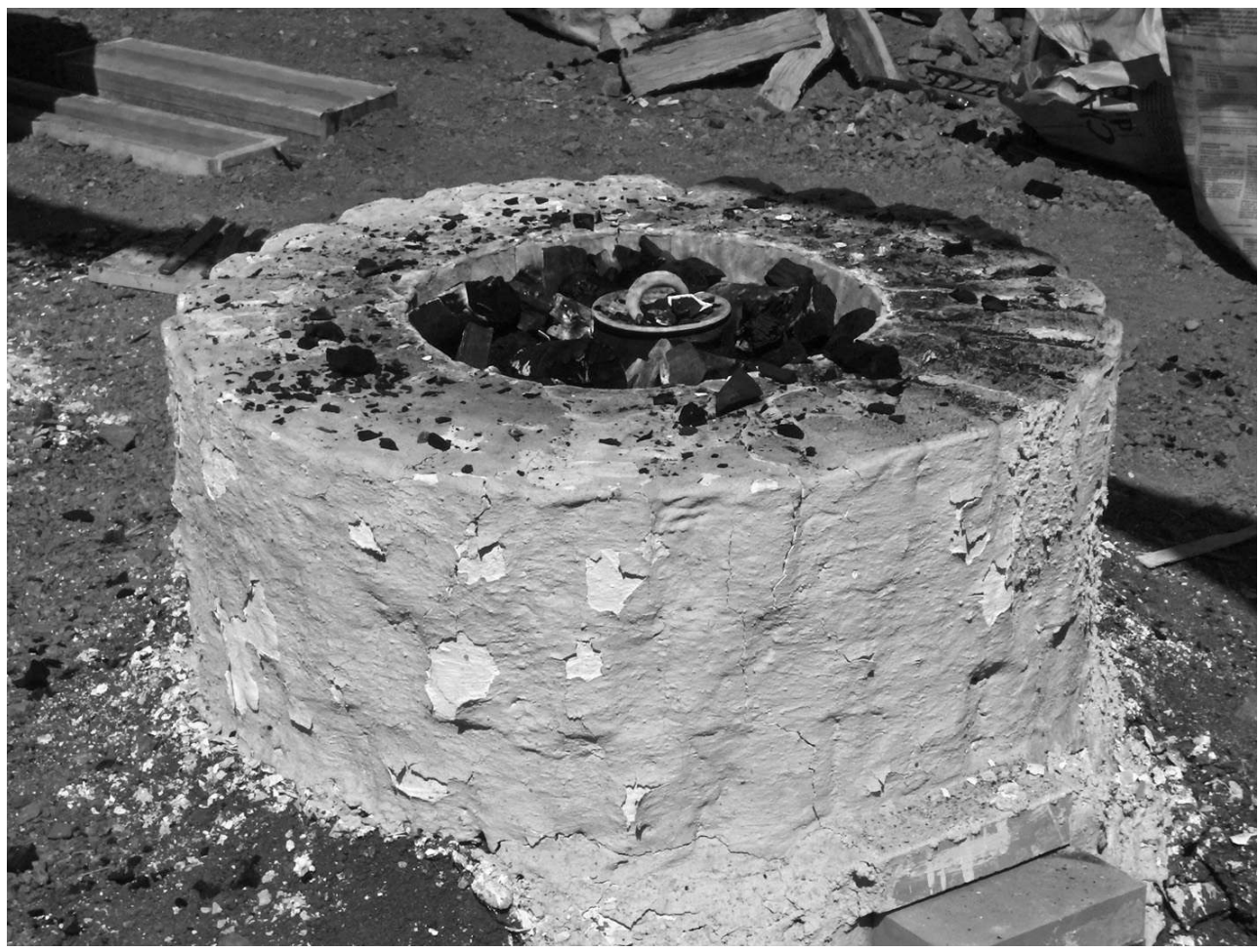

27 - Un four en trou de serrure (un four médiéval du XIV e siècle apr. J.-C.) a été utilisé pour des raisons pratiques évidentes et faute de données historiques corrélées au 
monnayage étudié ici (fig. 5). La conception relativement simple de cette structure permet d'atteindre des températures élevées uniquement par tirage naturel. Nous pouvons aussi observer les différents changements qui ont lieu dans le foyer lors de l'opération de refonte (la flamme change de couleur, un dépôt jaune se forme sur les charbons, le creuset change de couleur, etc.). observe dans les monnaies antiques. Les analyses par activation avec des neutrons rapides de cyclotron ont en effet montré qu'il y a au maximum $25 \%$ de zinc dans les monnaies d'Auguste (Gaffiero, Suspène et al., à paraitre). Notre hypothèse de départ était qu'après quelques refontes nous devions arriver à un alliage à $25 \%$ de zinc. Les coulées et refontes ont été effectuées sur la plateforme expérimentale du site des Mines d'Argent des Rois Francs à Melle ${ }^{10}$ (Deux-Sèvres). couvercle en terre cuite est mis dans le foyer du four. Il est disposé au fond du foyer, entouré de braises jusqu'à le recouvrir. Pour les premières refontes une température de $900{ }^{\circ} \mathrm{C}$ est nécessaire pour amener le métal à l'état liquide; par conséquent, la température du foyer devant être d'une centaine de degrés Celsius supérieure au point de fusion de l'alliage afin que la coulée soit techniquement possible, le foyer doit être porté à $1000^{\circ} \mathrm{C}$. Lorsqu'il est bien entretenu le foyer atteint des températures supérieures à $1000^{\circ} \mathrm{C}$ : les refontes sont donc envisageables.

ant 20 à 30 minutes nous vérifions que le creuset est bien recouvert de braises et de charbons. Au bout de 20 minutes les charbons se colorent en jaune, le creuset devient d'une couleur rouge vif: il a une température de l'ordre de $1000^{\circ} \mathrm{C}$. Quelques minutes plus tard, on voit des fumées jaunes qui se dégagent du creuset: le laiton est alors à l'état liquide et est prêt pour la coulée. Il ne reste plus qu'à dégager les braises et charbons qui se trouvent sur le creuset et à le sortir du four à l'aide d'une pince et à verser le laiton dans le moule. La refonte est effectuée, il ne reste plus qu'a attendre que les moules refroidissent. Une bonne demi-heure est nécessaire avant de les ouvrir.

34 Le protocole qui a été suivi lors des refontes peut être résumé comme suit.

1) Préchauffage des moules pendant 1 heure minimum.

2) Assemblage du moule bivalve dans l'étau.

3) Mise au four du creuset rempli de laiton et recouvert d'un couvercle dans le four pendant environ 20 minutes selon la température du four.

4) Coulée du métal dans le moule : la température du four est supérieure à $1200^{\circ} \mathrm{C}$ au moment de la fonte.

5) Le creuset est sorti du four pour lui permettre de refroidir ; récupération du laiton déposé sur les parois.

6) Attente de 30 minutes à 1 heure pour que le moule refroidisse. 
7) Ouverture et photographie des moules et des flans.

8) Découpage des flans pour la pesée.

9) Pesée et prélèvements en début et fin de coulée.

10) Remise du creuset et du métal restant au four.

11) La coulée suivante est prête.

Les refontes (fig. 6) ont permis l'obtention de diverses grappes de flans (fig. 7).

Figure 6 : Coulée du laiton (site des mines d'argent des rois francs de Melle, Deux Sèvres) Figure 6: Melting of the brass (site of the silver mines of the Frank kings in Melle, Deux-Sèvres).

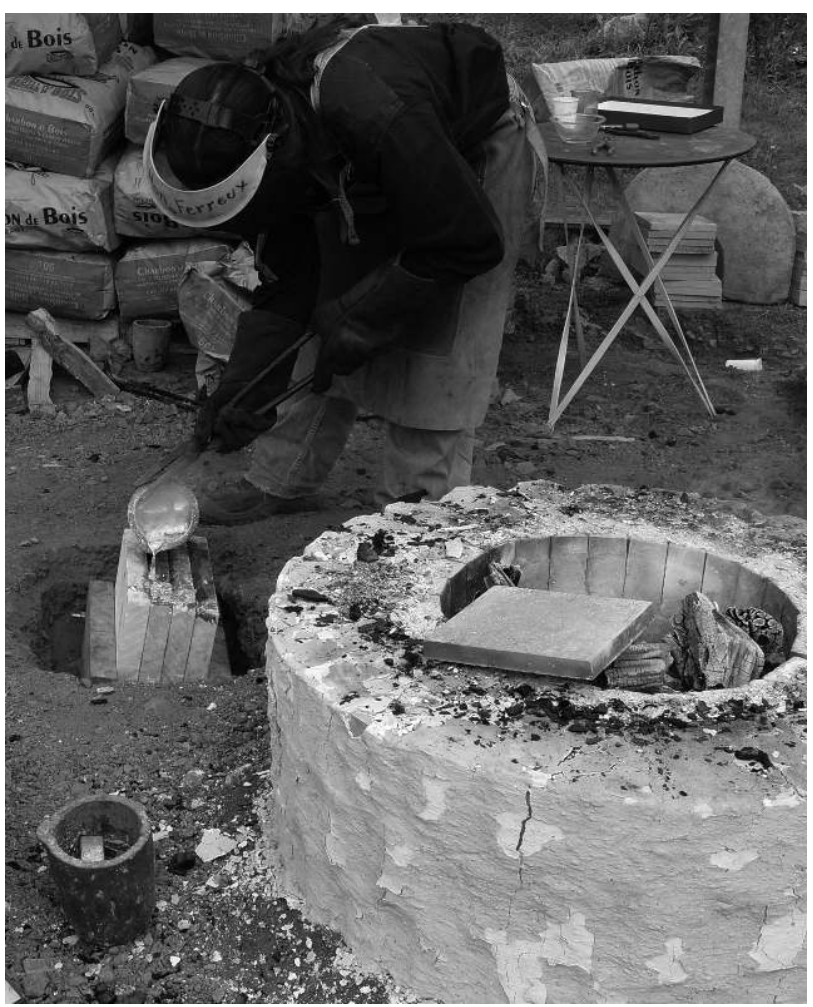


Figure 7 : Chapelet de flans en orichalque. Figure 7: Stringer of orichalcum's blanks.

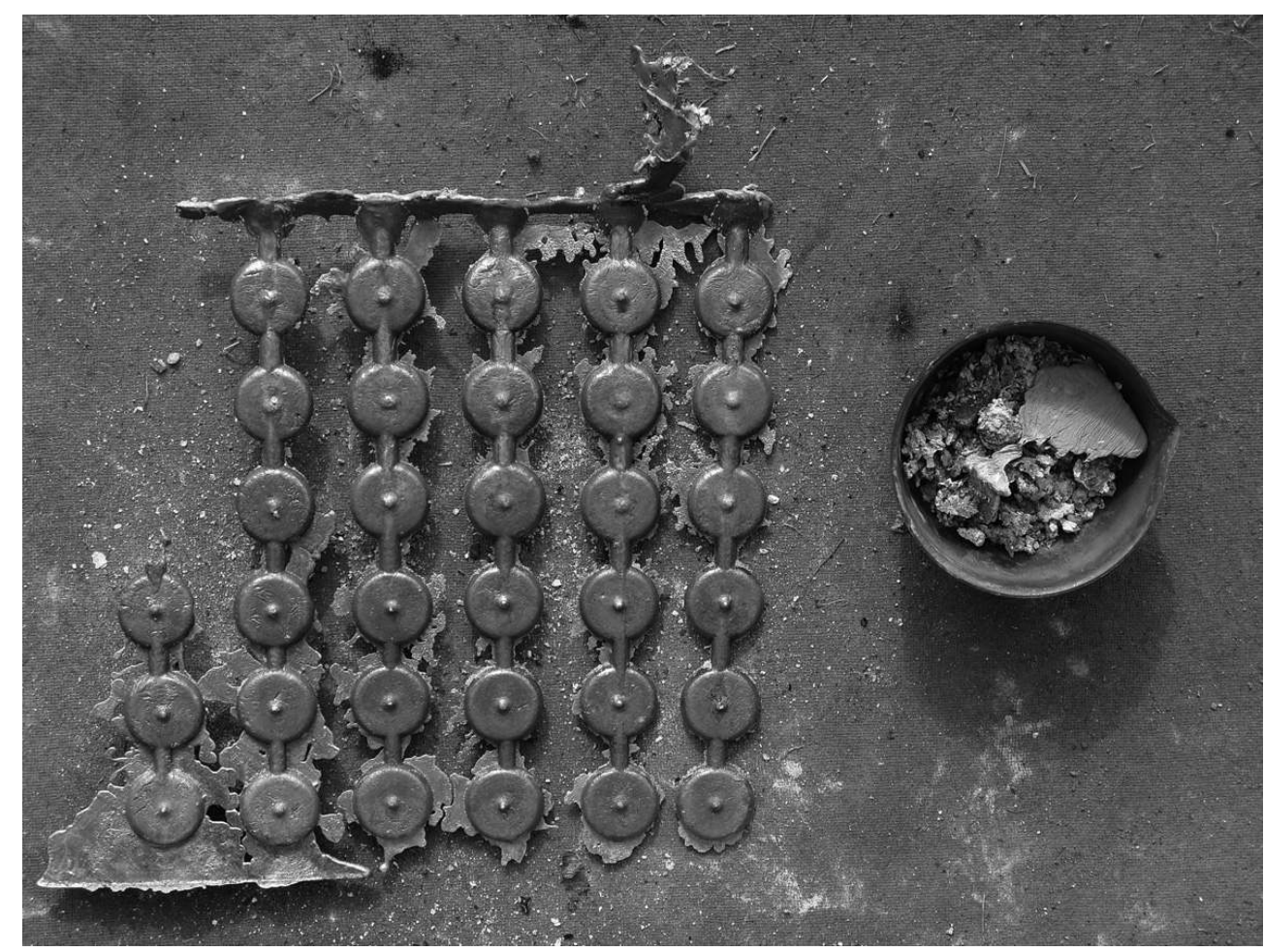

36 Après prélèvements ${ }^{11}$ pour analyse élémentaire, les flans ont été refondus une vingtaine de fois afin de simuler le phénomène de refontes monétaires.

\section{Les résultats obtenus}

37 À l'issue des expérimentations, la courbe des refontes montre une diminution lente, d'allure quasi linéaire, de la teneur en zinc en fonction du nombre de refontes (fig. 8). À chaque refonte, la perte s'élève à 1,5\% de la teneur en zinc précédente. 
Figure 8 : Évolution de la teneur en zinc selon le nombre de refontes et courbe de tendance exponentielle.

Figure 8: Evolution of the zinc content according to the number of remeltings and curve with an exponential tendency.

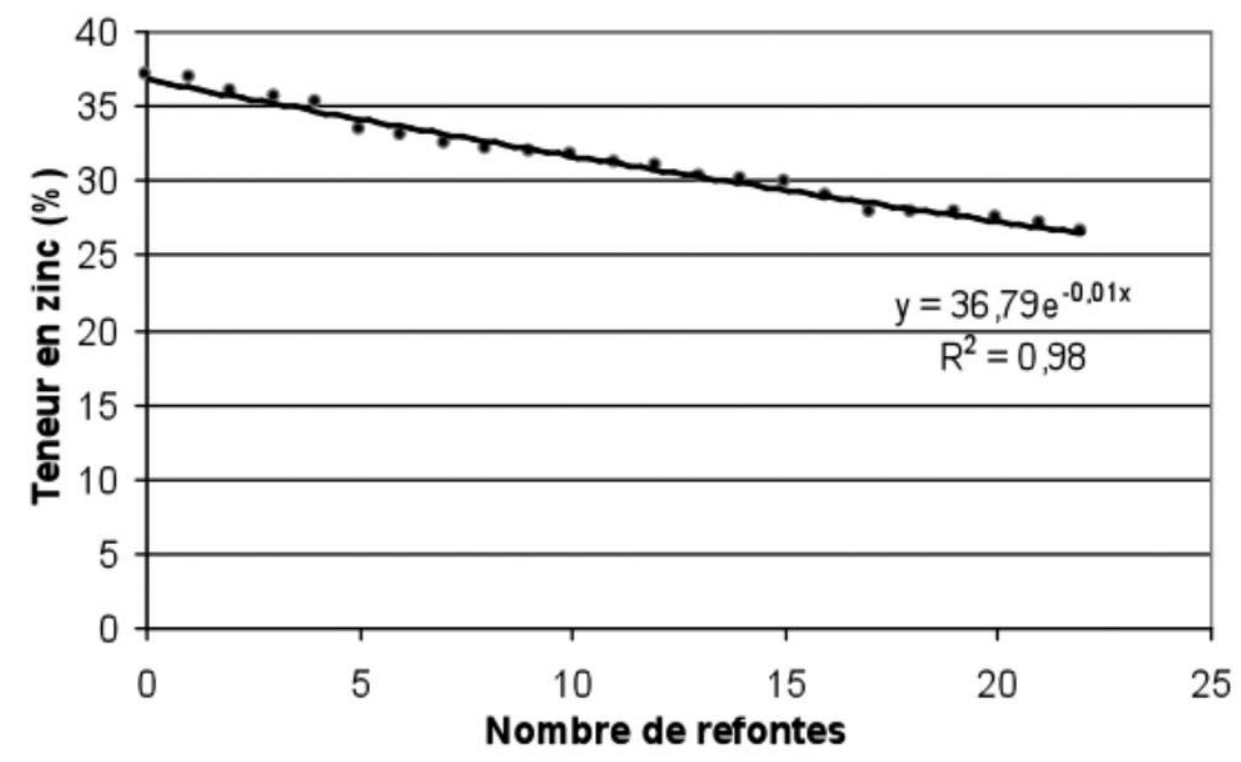

Contrairement à notre hypothèse initiale, l'alliage contenait encore $26 \%$ de zinc après 22 refontes. Nous pensions en effet que malgré l'utilisation d'un couvercle la perte en zinc serait plus importante. Si la température de fusion du zinc est de $420^{\circ} \mathrm{C}$ tandis que celle du cuivre est de $1084^{\circ} \mathrm{C}$, celle qui correspond à un alliage des deux (5 à $30 \%$ de zinc) est comprise entre $910^{\circ} \mathrm{C}$ et $1080^{\circ} \mathrm{C}$. Cette gamme de température est bien supérieure à la température de fusion du zinc et dépasse sa température de vaporisation $\left(907^{\circ} \mathrm{C}\right)$; c'est donc bien cet élément qui partira préférentiellement lors des refontes (Baïlon et Dorlot, 2000, p. 527-530). Dans le four électrique du laboratoire, nous avions uniquement recouvert l'alliage de charbon, qui n'a pas empêché la réaction d'oxydoréduction de se poursuivre. Sans couvercle, nous avions obtenu en laboratoire une diminution nette de $5 \%$ de zinc en 2 refontes.

Les températures du four électrique sont constantes et comprises entre $950^{\circ} \mathrm{C}$ et 1 $150{ }^{\circ} \mathrm{C}$ (moins il y a de zinc, plus la température du four a été augmentée). Celles du four expérimental varient beaucoup plus et sont très sensibles aux conditions météorologiques.

Dans le foyer au niveau de la sole, la température est la plus haute, elle peut ponctuellement atteindre jusqu'à $1400^{\circ} \mathrm{C}$. Plus on se rapproche de la surface et de l'air extérieur, plus la température diminue. Dans le creuset, nous n'avons pas mesuré la température pour éviter d'ouvrir le couvercle et de laisser plus d'air entrer : on voulait éviter la réaction d'oxydoréduction, mais nous pouvons estimer qu'elle devait être du même ordre de grandeur que celle de la fusion de l'alliage.

41 Si la température varie plus lors des expérimentations réalisées dans le four en trou de serrure que lors de celles faites dans four électrique, le contrôle de la fusion de l'alliage et la maitrise du temps nécessaire à cette dernière est cependant plus facile avec le four à trou de serrure. Cela explique que la perte de métal ait été plus facile à contrôler et à minimiser lors des expérimentations que lors des essais effectués au laboratoire. 

ne peut être uniquement la conséquence d'une perte accidentelle de l'élément zinc. Elle monétaires. L'utilisation de couvercle est attestée dans de multiples sites métallurgiques (Pernot, 1999), et nous pouvons donc supposer que cet objet était employé dans les ateliers monétaires romains. Le couvercle qui était essentiellement utilisé d'un point de vue pratique lors de notre expérimentation empêche les charbons de tomber dans le creuset, ce qui facilite aussi la coulée. Le zinc ne rentrera ainsi en contact qu'avec une quantité réduite d'oxygène (l'oxygène présent dans le creuset au début de l'expérimentation), ce qui limitera son oxydation, et donc sa perte. Le métal étant une matière première recherchée, qui nécessite un certain nombre de traitements (le minerai est extrait, puis traité afin d'être prêt à l'emploi, pour finalement être transformé selon les besoins), on peut imaginer que les artisans métallurgistes devaient d'autant plus limiter les pertes de métal. De plus, le a rationibus ${ }^{12}$ contrôlant la production monétaire dans l'atelier, on peut supposer que les pertes métalliques étaient surveillées. Il devait en effet évaluer la quantité de monnaies à émettre, et donc indirectement estimer la quantité nécessaire de métal à employer (Burnett, 1988).

L'hypothèse de refontes non contrôlées est donc à écarter, car un minimum de précautions permet de contrôler et de minimiser la perte en zinc. La diminution de la teneur en zinc dans les monnaies romaines pourrait donc s'expliquer par d'autres théories. Une action volontaire de l'autorité émettrice par souci d'économie, par manque de minerais de zinc, de laiton, ou encore, pour conserver les équivalences entre les différentes espèces lors des affaiblissements du denier, est envisageable. La baisse des teneurs en zinc pourrait aussi être expliquée par l'ajout de cuivre à l'alliage riche en zinc. De ce fait on diminue relativement la teneur en zinc tout en augmentant la masse monétaire à frapper. On obtiendrait alors le même type de courbe. Il pourrait cependant y avoir des changements au niveau des éléments-traces si l'on ajoute un cuivre différent du cuivre initial (cet aspect est en cours d'étude). La courbe de la figure 2 montre que les écart-types sur la teneur en zinc sont importants, ceci est dû à la forte dispersion des teneurs en zinc dans les monnaies. La couleur jaune du laiton est probablement un des critères visuels lors de la fabrication de l'alliage ou des monnaies. Or, pour des teneurs en zinc comprises entre 10 et $25 \%$, cet aspect visuel change peu et n'est pas facilement décelable pour un œil non averti. Il est donc possible d'ajouter du cuivre sans pour autant dévaluer la monnaie. La difficulté de contrôler le titre d'un laiton est due à la nature particulière de cet alliage. Contrairement aux autres alliages 
(bronze, billon...) obtenus par mélange de métaux, la fabrication du laiton se rapproche plus à l'époque d'un procédé de coloration du cuivre par mélange avec une terre que de ceux utilisés pour la réalisation d'un alliage métallique. Le métallurgiste se base ainsi probablement sur la couleur du produit obtenu et non pas sur les quantités de métaux employés qui hormis celle du cuivre lui sont inconnues. La quantité de zinc ajouté dépend en effet de la nature et de la pureté des oxydes de zinc employés. Par contre, l'apparition de plomb et d'étain à partir d'Hadrien (Caley, 1964) implique des ajouts, qui font diminuer la teneur en zinc. L'ensemble de ces hypothèses seront étudiées ultérieurement.

\section{Conclusion}

Les études de compositions chimiques rassemblées dans l'ouvrage de E. Caley dans les années soixante montrent une diminution de la teneur en zinc. Une première hypothèse pour expliquer cette tendance est que la baisse serait due au réemploi des monnaies des empereurs précédents, elles seraient refondues pour servir de matière première aux nouvelles monnaies. Les analyses élémentaires ont aussi montré l'apparition de plomb et d'étain dans les pièces tardives (à partir d'Hadrien), ce qui sous entend un ajout de métal en plus de refontes potentielles. En 1996, D. Dungworth reprend ces travaux en regroupant de nouvelles analyses de monnaies en orichalque et montre théoriquement que la baisse de la teneur en zinc ne peut être uniquement due aux refontes, il y a aussi des ajouts de cuivre, voir de plomb et d'étain dans l'alliage monétaire.

Depuis 2007, nous avons décidé de reprendre à notre tour cette étude, en analysant cette fois-ci un plus grand corpus monétaire à l'aide d'une même méthode d'analyse non destructive (l'ANRC) afin de vérifier si la variation de la teneur en zinc dans les monnaies est semblable à celle obtenue avec différentes méthodes d'analyse de composition.

Il apparait que la diminution des teneurs en zinc commence plus tardivement que ce que E. Caley a pu observer: elle se manifeste en réalité seulement après la mort de Néron. D'après la courbe (Figure 2) la diminution de la teneur en zinc est surtout sensible à partir de Vespasien; on peut envisager des refontes conséquentes à partir de cet empereur. Nous avons donc mis en place une série d'expérimentations métallurgiques portant sur les refontes afin de mieux comprendre ce principe et de tester leur influence sur la teneur en zinc de l'alliage.

Cette première série d'expérimentations métallurgiques, réalisées à Melle, nous permet de nuancer l'influence des refontes sur l'évolution de la teneur en zinc du monnayage romain: un artisan métallurgiste semble capable de contrôler les pertes de métal et donc de zinc en utilisant un simple couvercle. La diminution de la teneur en zinc ne peut être accidentelle. Elle est minimisée (les pertes dues à la réaction d'oxydoréduction avec l'oxygène présent dans le creuset sont inévitables). De plus, en utilisant au préalable un four électrique pour effectuer les refontes, il est clairement apparu que l'alliage réagit différemment selon le four choisi. Les résultats obtenus à Melle montrent que voir le creuset à l'intérieur du four permet de mieux gérer les temps de fontes et de déterminer le meilleur moment pour couler le métal.

Les hypothèses portant sur l'utilisation d'un nouveau laiton ou sur l'ajout de cuivre peuvent aussi expliquer cette baisse. Ajouter du cuivre fait certes baisser la teneur en 
zinc, mais permet également de frapper plus de monnaies. Une combinaison de refontes (au moins ponctuelles) et d'ajouts est aussi possible puisqu'il y a quelques mentions de refontes sur le monnayage de Caligula et sous Trajan.

51 Enfin, l'utilisation de plomb et d'étain à partir d'Hadrien n'a pas été abordée ici, mais elle relativise aussi l'influence des refontes sur l'alliage monétaire.

Par la suite nous devrons vérifier que l'élément zinc n'est pas plus instable dans cette gamme de teneurs, et pour cela nous allons procéder à de nouvelles refontes en partant de $25 \%$ au lieu de $37 \%$ de zinc. En effet, lors de nos expérimentations, les teneurs en zinc obtenues n'ont pas atteint le niveau de celles que l'on trouve dans le monnayage en orichalque romain (entre 25 et $5 \%$ ). Une perte massive de zinc liée aux seules refontes semble toutefois peu vraisemblable.

Nous remercions M. Amandry, directeur du Cabinet des Médailles de la Bibliothèque nationale de France, et D. Hollard, conservateur au Cabinet des médailles de la Bibliothèque nationale de France, de nous avoir permis d'analyser les monnaies romaines en orichalque, et de nous avoir fait bénéficier de leurs conseils et de leur expérience. Nous remercions l'équipe de la plateforme expérimentale du site des Mines d'Argent des Rois Francs de Melle (Deux-Sèvres) pour son accueil, sa disponibilité et son aide. Nous remercions aussi le CEMTHI pour nous avoir accordé un accès privilégié au cyclotron, ainsi que la Région Centre qui nous a octroyé une bourse de thèse pour le financement de ces recherches.

\section{BIBLIOGRAPHIE}

BAÏLON, J.-P. et DoRLOT, J.-M., 2000. Des Matériaux, Montréal, Presses internationales polytechnique.

BARRANDON, J.-N., SUSPÈNE, A. et GAFFIERO, A., à paraître. « Les émissions d'as au type Divvs Avgvstvs Pater frappées sous Tibère : L'apport des analyses à leur datation et à leur interprétation », Revue Numismatique (sous presse).

BEAUCHESNE, F. 1986. Analyse non destructive du cuivre et de ses alliages par activation à l'aide de neutrons rapides de cyclotron. Applications à la numismatique, Thèse de doctorat, Université d'Orléans, France.

BESOMBES, P.-A., 2008. Monnaies de l'Empire romain IV Trajan (98-117 après J.-C.), Paris/Strasbourg, Bibliothèque nationale de France.

BURNETT, A., 1988. La numismatique romaine - De la République au Haut-Empire, Paris, Errance.

CALEY, E., 1964. Orichalcum and related ancient alloys : origin, composition and manufacture, with special reference to the coinage of the Roman Empire, New York, American Numismatic Society, Numismatic notes and monographs, vol. 151.

CAllu, J.-P., BRENOT, C., BARRANDON, J.-N. et POIRIER J., 1985. « Aureus Obryziacus », in Morrisson C., Brenot C., Callu J.-P., Barrandon J.-N., Poirier J., Halleux R. (dir). L'or monnayé I, Purification et altération de Rome à Byzance, Cahiers Ernest-Babelon 2, Paris, CNRS édition, 81-111. 
Christol, M. et NonY, D., 2003. Rome et son empire, Paris, Hachette Supérieur.

Dungworth, D., 1996. Caley's ‘zinc decline' reconsidered. The Numismatic Chronicle, Vol. 156, p. 228-234.

ÉTIENNE, R. et RACHET, M., 1984. Le trésor de Garonne - Essai sur la circulation monétaire en Aquitaine à la fin du règne d'Antonin le Pieux (159-161), Bordeaux, Fédération historique du Sud-ouest.

GAFFIERO, A., SUSPÈNE, A., TÉREYGEOL, F. et GRATUZE, B., à paraître. Le monnayage en orichalque romain : apport des expérimentations aux études numismatiques, in Actes du Congrès International de Numismatique de Glasgow 2009.

Pernot, M., 1999. L'artisanat, in Chardron-Picault, A. et Pernot, M. (dir.). L'artisanat. Un quartier antique d'artisanat métallurgique à Autun. Le site du Lycée militaire, D. d'A. Française, 76, p. 153-189.

Pilon, F., 2002. La fabrication de monnaies d'imitation à Châteaubleau (Seine-et-Marne, France) au III siècle apr. J.-C., in Auberson, A. F., Derschka, H. R. et Frey-Kupper, S. (dir.). Étude de numismatique et d'histoire monétaire 5. Faux - contrefaçons - imitations, Éditions du Zèbre, p. 43-53.

WALKER, D. R., 1976. The metrology of the Roman silver coinage. Part 1 : From Augustus to Domitian. British Archaeological Reports, Supplementary Series, Number 5. British Archaeological Reports, Oxford, UK.

WALKER, D. R., 1977. The metrology of the Roman silver coinage. Part 2 : From Nerva to Commodus, British Archaeological Reports, Supplementary Series, Number 22, British Archaeological Reports, Oxford, UK.

WALKER, D. R., 1978. The metrology of the Roman silver coinage, Part 3 : From Pertinax to Uranius Antoninus, British Archaeological Reports, Supplementary Series, Number 40, British Archaeological Reports, Oxford, UK.

\section{NOTES}

1. Le zinc devant être ajouté au cuivre sous forme de minerai pour élaborer le laiton, il nécessite l'emploi de charbon ainsi que de matériels supplémentaires lors des opérations de cémentation.

2. La partie irradiation avec des neutrons rapides de cyclotron à été effectuée au CEMTHI (CNRS Orléans). La partie détection par spectrométrie gamma ainsi que traitement des données ont eu lieu au laboratoire IRAMAT (CNRS Orléans).

3. Les différents types de minerai peuvent amener à distinguer des émissions non datées entre-elles et permettent de proposer à l'aide des éléments-traces des émissions datées une date d'émission: Barrandon et al. à paraitre. Les sources de minerai ne sont pas distinguées en revanche.

4. Gaffiero, A. thèse en cours portant sur «Le monnayage en orichalque de l'Empire romain : de la réforme d'Auguste à Commode».

5. Les monnaies analysées par activation avec des neutrons rapides de cyclotron proviennent essentiellement du Cabinet des médailles de la Bibliothèque nationale de France.

6. L'analyse par activation avec des neutrons rapide de cyclotron se notera ANRC par la suite. 
7. Une à cinq monnaies sont analysées par empereur.

8. Seule une approche statistique fondée sur la quantification des coins monétaire ainsi que sur les différents trésors retrouvés nous permet d'évaluer la taille probable des émissions.

9. Dion Cassius, LX, 22.

10. Opération conduite dans le cadre du Programme Collectif de Recherche "paléométallurgie et expérimentation » de la DRAC-SRA Poitou-Charentes.

11. Pour chacune des coulées, nous effectuons deux prélèvements: le premier flan coulé, ainsi que l'un des derniers flans sont sélectionnés.

12. A rationibus : il s'agit du responsable des finances, selon Pline (EP. 8.6) c'était le "gardien de la richesse impériale ».

\section{RÉSUMÉS}

Les monnaies d'orichalque (un alliage de cuivre et de zinc aussi appelé laiton) sont émises de façon systématique sous le principat d'Auguste à partir de 23 av. J.-C. Ce monnayage est composé de sesterces, de dupondii et de semis. Des analyses élémentaires effectuées sur ces monnaies par spectrométrie de fluorescence $\mathrm{X}$, par activation avec des neutrons rapides de cyclotron et par voie humide ont montré que la composition de l'alliage monétaire évolue au cours des émissions : la teneur en zinc diminue. Une des hypothèses émises est que cette baisse serait liée aux refontes successives des monnaies lors de la fabrication de nouvelles émissions.

Orichalcum coins (an alloy of copper and zinc also named brass) are systematically issued during the Principate of Augustus from 23 B. C. on. These coins are sesterstii, dupondii and semisses. Elementary analysis of these coins by X-ray Fluorescence spectroscopy, by activation with fast neutrons from a cyclotron and by chemical analysis show an evolution of the alloy composition during the issues: the zinc content decreased. An hypothesis is that this decrease is related to successive meltings of the coins for the elaboration of new issues.

\section{INDEX}

Mots-clés : expérimentation, flans, monnaie, orichalque, refonte

Index chronologique : Antiquité / Antiquity

Keywords : blanks, coin, experimentation, orichalcum, melting 


\section{AUTEURS}

\section{GAFFIERO}

IRAMAT, Centre Ernest-Babelon, UMR 5060, CNRS, - Université d'Orléans, 3 D rue de La Férollerie, 45071 Orléans cedex 2. (arwen.gaffiero@wanadoo.fr), (suspene@yahoo.fr),

(gratuze@cnrs-orleans.fr

\section{FLORIAN TÉREYGEOL}

IRAMAT, Laboratoire « Métallurgie et Culture », UMR 5060, CNRS, 90010 Belfort, UMR 9956 Laboratoire Pierre-Süe, CEA Saclay, 91191 Gif-sur-Yvette Cedex. (florian.tereygeol@cea.fr)

\section{A. SUSPÈNE}

IRAMAT, Centre Ernest-Babelon, UMR 5060, CNRS, - Université d'Orléans, 3 D rue de La Férollerie, 45071 Orléans cedex 2. (arwen.gaffiero@wanadoo.fr), (suspene@yahoo.fr), (gratuze@cnrs-orleans.fr

\section{BERNARD GRATUZE}

IRAMAT, Centre Ernest-Babelon, UMR 5060, CNRS, - Université d'Orléans, 3 D rue de La Férollerie, 45071 Orléans cedex 2. (arwen.gaffiero@wanadoo.fr), (suspene@yahoo.fr), (gratuze@cnrs-orleans.fr

\section{S. ZELLER}

Étudiante en Master 2 d'Archéologie des Périodes Historiques à l'université de Paris I PanthéonSorbonne. (stephanie.zeller@voila.fr) 